\title{
Differential maturation trajectories of innate antiviral immunity in health and atopy
}

\author{
Anastasia Georgountzou ${ }^{1}$, Dimitra Kokkinou ${ }^{1}$, Stella Taka ${ }^{1}$, Paraskevi Maggina ${ }^{1}$, John \\ Lakoumentas $^{1}$, Vassiliki Papaevangelou ${ }^{1}$, Maria Tsolia ${ }^{1}$, Paraskevi Xepapadaki ${ }^{1}$, Evangelos \\ Andreakos $^{2}$, and Nikos Papadopoulos ${ }^{1}$ \\ ${ }^{1}$ National and Kapodistrian University of Athens \\ ${ }^{2}$ Biomedical Research Foundation of the Academy of Athens
}

January 18, 2021

\begin{abstract}
Background The maturation of innate immune responses in health and atopy is still incompletely understood. Methods We aimed to evaluate age-related trajectories of the TLR3 and TLR7/8 pathways across the lifespan and whether these differ between healthy and atopic individuals. Peripheral blood mononuclear cells (PBMCs) were isolated from 39 otherwise healthy atopic and 39 non-atopic subjects, aged 0-45 years. Selected cytokines involved in antiviral responses were measured by Luminex in culture supernatants of poly(I:C)- and R848-stimulated PBMCs. The non-parametric correlation between age and cytokine expression and differences in developmental trajectories between healthy and atopic were estimated. Patterns of cytokine development were identified with principal component analysis. Results Normal innate immune maturation entails significant and progressive age-related changes in the production of IL-1 $\beta$, TNF- $\alpha$, MIP-1 $\beta$, MCP-3, IP-10, IL-10, IL-12p70 and IFN- $\gamma$ upon TLR3 and/or TLR7/8 stimulation. Individual cytokines made small contributions to the observed variability; chemokines MCP-3 and IP-10 were key contributors. The development of these pathways deviated in atopic subjects with significant differences observed in the trajectories of IL- $1 \beta$, MIP- $1 \beta$ and IL-10 synthesis. Conclusion TLR3 and TLR7/8 pathways mature during childhood, while atopy is associated with an abnormal maturation pattern. Suboptimal responses in Th1, inflammatory cytokine and chemokine production may be implicated in poor antiviral immunity in atopics, while deficient maturation of IL-10 producing capacity in the breaking of tolerance.
\end{abstract}

\section{Hosted file}

MANUSCRIPT_PAI.pdf available at https://authorea.com/users/390194/articles/504605differential-maturation-trajectories-of-innate-antiviral-immunity-in-health-and-atopy 

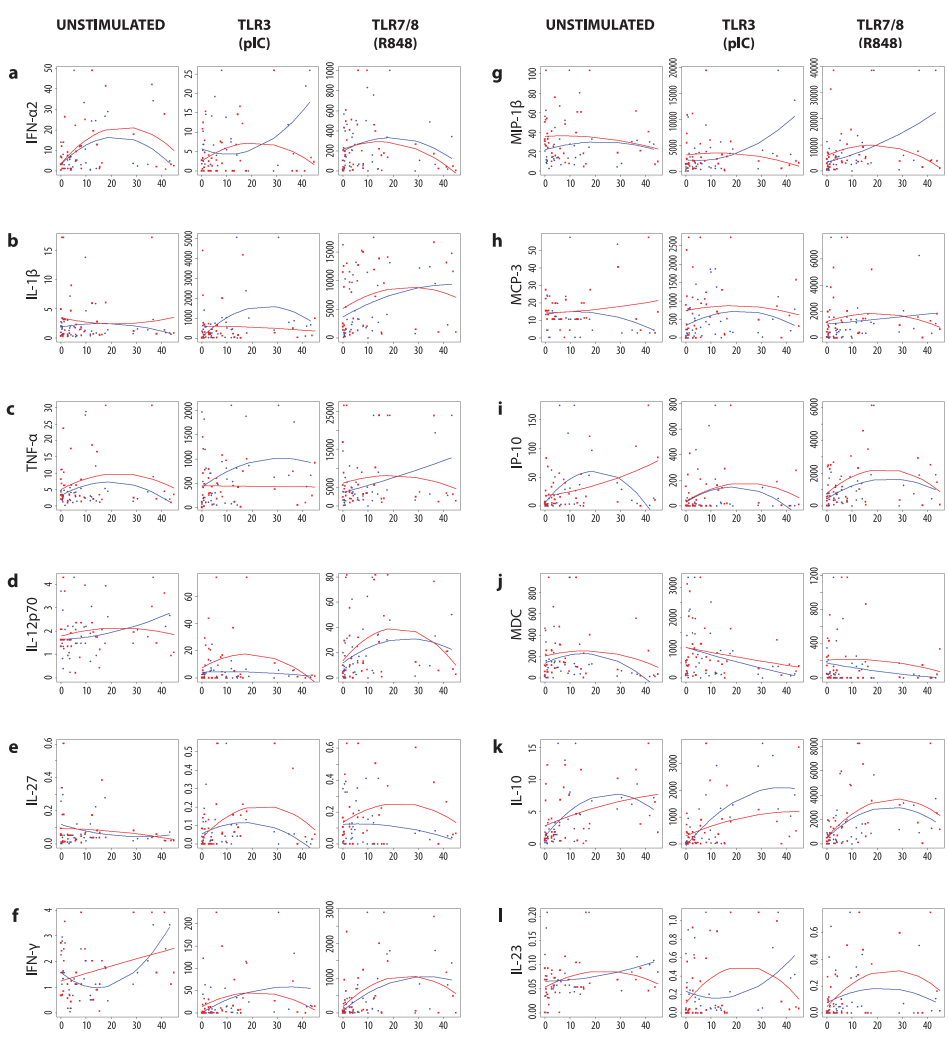

AGE

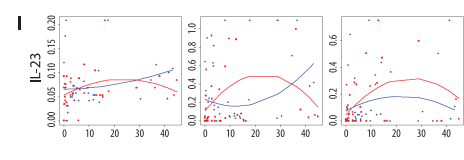

AGE

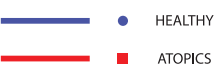




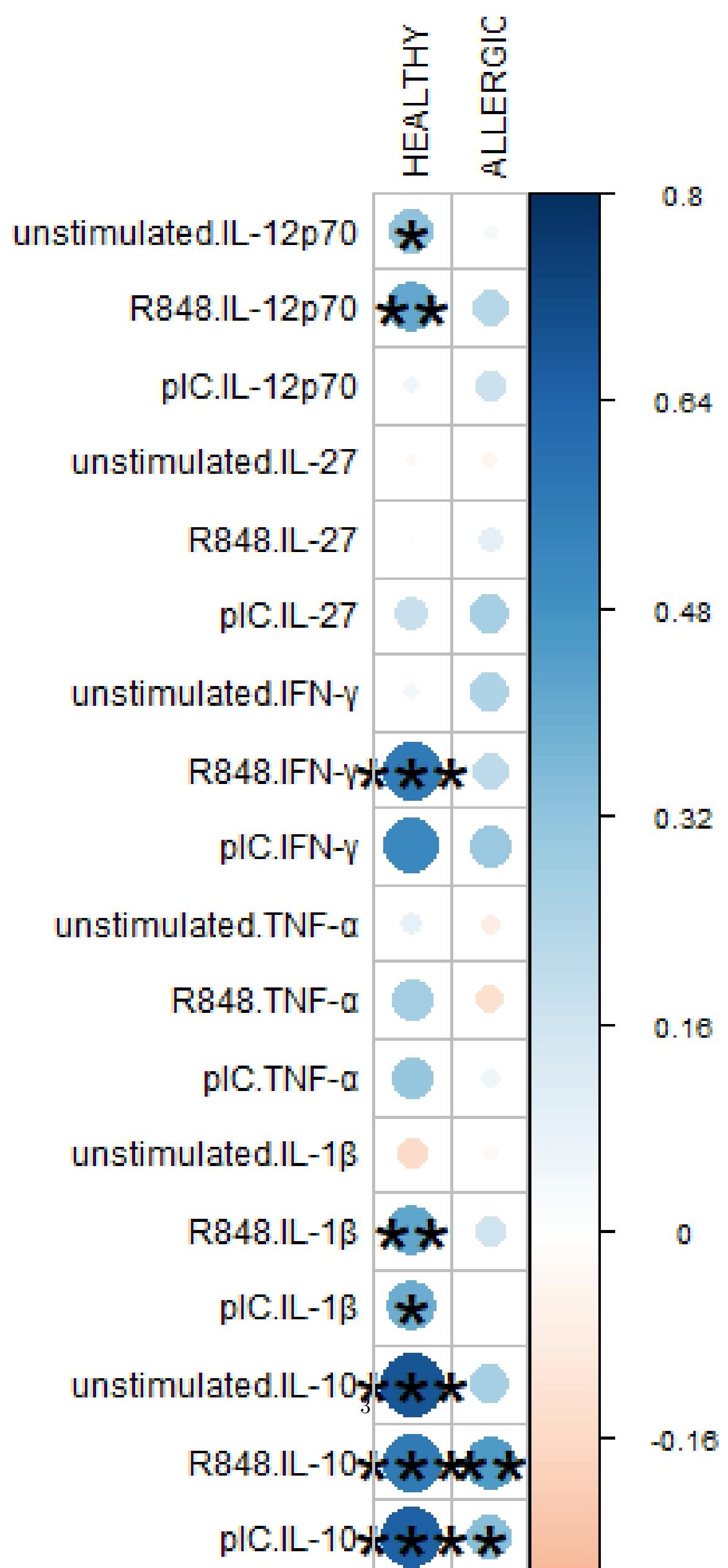



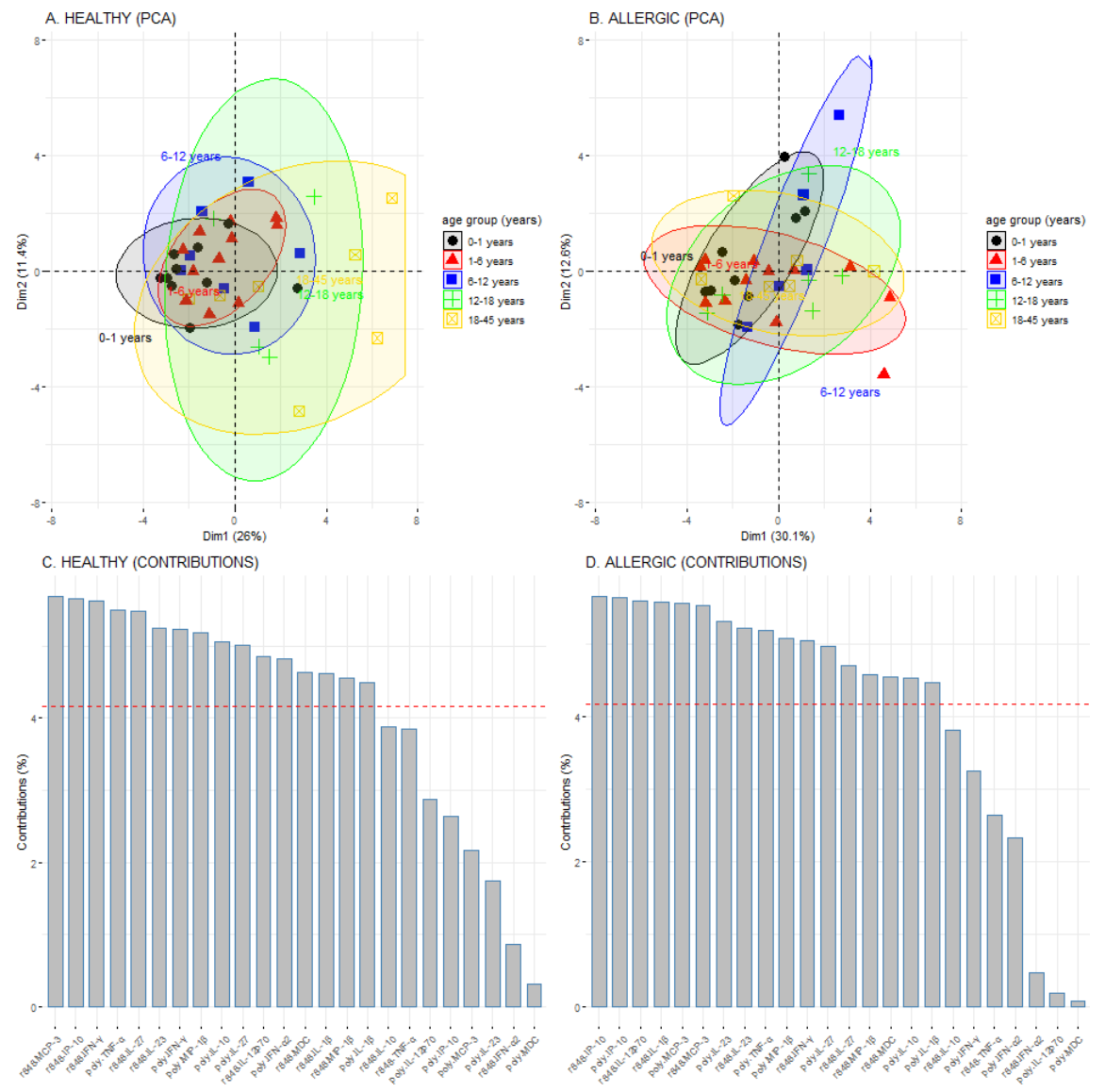